\title{
Local Control Strategies for Groups of Mobile Autonomous Agents
}

\author{
Zhiyun Lin, Mireille Broucke, Member, IEEE, and Bruce Francis, Fellow, IEEE \\ Department of Electrical and Computer Engineering \\ University of Toronto \\ Toronto, Canada, M5S3G4 \\ linz, broucke, francis@control.utoronto.ca
}

\begin{abstract}
The problem is studied of achieving a specified formation among a group of mobile autonomous agents by distributed control. If convergence to a point is feasible, then more general formations are achievable too, so the focus is on convergence to a point (the agreement problem). Three formation strategies are studied and convergence is proved under certain conditions. Also, motivated by the question of whether collisions occur, formation evolution is studied.
\end{abstract}

\section{INTRODUCTION}

In 1987 Reynolds [16] introduced a model and wrote a program called boids [17] that simulates a flock of birds in flight; they fly as a flock, with a common average heading, and they avoid colliding with each other. Each bird has a local control strategy, yet a desirable overall group behavior is achieved. The local strategy of each bird has three components: separation, steer to avoid crowding; alignment, steer towards the average heading of neighbors; cohesion, steer towards the average position of neighbors. Recently, Jadbabaie et al. [7] formulated a 2-dimensional version of Reynolds' setup and studied one of the steering strategies. They proved that the alignment strategy leads, under a certain assumption (the graph representing which agents are neighbors of another always is connected, or at least periodically connected), to the result that all the agents' headings converge to a common heading. Besides being of interest in biology, Reynolds' ideas have relevance in the subject of multiple vehicle formations, e.g., [20], [19], [15]. Generally, the objective is for a group of mobile agents (robot rovers, unmanned air vehicles, or unmanned underwater vehicles) either to achieve a formation, or to move while maintaining a formation, or to reconfigure from one formation to another.

Recently, several researchers have investigated issues in distributed algorithms for multi-agent systems. In [19], a group of simulated robots form approximations to circles and simple polygons, using the scenario that each robot orients itself to, e.g., the furthest and nearest robot. In [3], a similar setup is presented, but collision avoidance and group motion, e.g., a matrix formation performing a right turn, are also considered. And in [1], [13], [14], distributed algorithms are studied where a set of robots represented as points in the plane should converge to a point; this is termed an agreement problem [1]. Besides the objective of rendezvousing at a common point, convergence is important for another reason: If convergence to a point is feasible, then more general formations are achievable too, as we show. Other relevant recent references are [4], [5], [9], [11], [15], [21], [22].

In this paper we study the suitability of three formation strategies. The first is cyclic pursuit. Cyclic pursuit is interesting because it is decentralized and requires the minimum number of communication links ( $n$ links for $n$ agents) to achieve a formation. It is well known (e.g., [2]) that under this strategy the agents converge to a point. Motivated by the question of whether collisions occur, we also study formation evolution. We show that if the agents initially are arranged in a counterclockwise star formation or a clockwise star formation, then they are always so arranged, and therefore there is no collision. We also study a modified strategy, where an agent pursues the virtual displacement of another. We study the achievable formations in this case.

In the second and third formation strategies, each agent can sense only some neighbor agents; first the undirectedgraph case where if agent $i$ senses agent $j$, then $j$ senses $i$ (the sensor graph is undirected); then the general directedgraph case (the sensor graph is directed).

Our setup is extremely simple: An agent is a point in the complex plane with no kinematic constraints of motion. In future work the agents will be wheeled vehicles; [12] has a study of unicycles under cyclic pursuit.

\section{Cyclic Pursuit}

Consider $n$ ordered and numbered points, $z_{1}, \ldots, z_{n}$, in the complex plane. Each represents a freely mobile agent. We consider the local strategy where each agent pursues the next one in the order. Thus, the model is

$$
\dot{z}_{i}=z_{i+1}-z_{i}, i=1, \ldots, n-1 ; \quad \dot{z}_{n}=z_{1}-z_{n},
$$

which can be assembled into vector form $\dot{z}=A z$. The matrix $A$ has one eigenvalue at the origin, all others having negative real part. Consequently [2], for all initial locations of the agents, the centroid of the points $z_{1}(t), \ldots, z_{n}(t)$ is stationary and every $z_{i}(t), i=1,2, \ldots, n$ converges to this centroid.

Convergence to a common location is an instance of an agreement problem. Besides being of interest in its own right, if convergence to a point is achievable, then other formations are achievable by a simple modification, where 
each agent pursues a displacement of the next agent:

$\dot{z}_{i}=\left(z_{i+1}+c_{i}\right)-z_{i}, i=1, \ldots, n-1 ; \quad \dot{z}_{n}=\left(z_{1}+c_{n}\right)-z_{n}$.

The vector form is $\dot{z}=A z+c$. If the centroid of the points $c_{1}, \ldots, c_{n}$ is not at the origin, then the centroid of the agents moves off to infinity. To avoid this, we must assume that the centroid of the points $c_{1}, \ldots, c_{n}$ is at the origin. Then $c \in \mathcal{E}$, the eigenspace of $A$ corresponding to eigenvalues with negative real parts, and there is a unique $d \in \mathcal{E}$ such that $A d+c=0$. The next result follows easily.

Theorem 1: Assume the centroid of the points $c_{1}, \ldots, c_{n}$ is at the origin. Then for any initial positions of the agents, the centroid of the points $z_{1}(t), \ldots, z_{n}(t)$ is stationary and every $z_{i}(t)$ converges to this centroid displaced by $d_{i}$.

A simulation to achieve an equilateral triangle formation is shown in Fig. 1. The initial locations of the six agents are randomly produced and the displacement vector is

$c=(-5+j 5 \sqrt{3},-5+j 5 \sqrt{3}, 10,10,-5-j 5 \sqrt{3},-5-j 5 \sqrt{3})$.

Now we turn to formation evolution, motivated by the issue of collision avoidance. Consider $n$ distinct points $z_{1}, \ldots, z_{n}$, not all collinear; see Fig. 2. Let $z_{0}$ be their centroid and $r_{i}$ be the distance between $z_{i}$ and the centroid. Let $\alpha_{i}$ denote the counterclockwise angle from line $\vec{z}_{0} \vec{z}_{i}$ to line ${\overrightarrow{z_{0}}}_{i+1}$ for $i=1, \ldots, n-1$ and $\alpha_{n}$ denote the counterclockwise angle from line $\overrightarrow{z_{0} z_{n}}$ to line $\overrightarrow{z_{0} z_{1}}$.

Definition 1: The $n$ points are said to be arranged in a counterclockwise star formation if $r_{i}>0$ and $\alpha_{i}>0$ for all $i=1, \ldots, n$ and $\sum_{i=1}^{n} \alpha_{i}=2 \pi$. They are said to be arranged in a clockwise star formation if $r_{i}>0$ and $\alpha_{i}<0$ for all $i=1, \ldots, n$ and $\sum_{i=1}^{n} \alpha_{i}=-2 \pi$.

In what follows, we consider only counterclockwise star formations, since clockwise star formations require an analogous treatment. Also, the case $n=2$ is trivial (the agents move in a straight line toward each other), so is omitted. Our main result is as follows.

Theorem 2: Suppose $n>2$ distinct points initially are arranged in a counterclockwise star formation. Under cyclic pursuit they remain in a counterclockwise star formation. (In particular, they never collide).

The proof requires some preliminary lemmas, whose proofs can be found in [10]. The first lemma is a tool for studying angles.

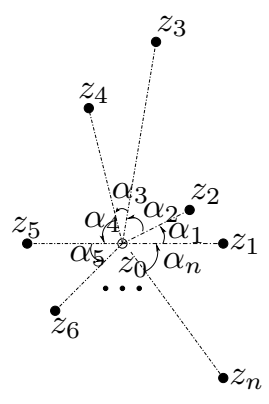

Fig. 2. A counterclockwise star formation.

Lemma 1: Let $z_{1}, z_{2}, z_{3}$ be three points in the complex plane, as shown in Fig. 3. Let $\alpha$ denote the counterclockwise angle from line $\overrightarrow{z_{2} z_{1}}$ to line $\overrightarrow{z_{2} z_{3}}, r_{1}=\left|z_{1}-z_{2}\right|$ and $r_{3}=\left|z_{3}-z_{2}\right|$. Define $F=\Im\left\{\overline{\left(z_{1}-z_{2}\right)}\left(z_{3}-z_{2}\right)\right\}$. Then

(a) $0<\alpha<\pi, r_{1}>0$, and $r_{3}>0$ iff $F>0$,

(b) $\pi<\alpha<2 \pi, r_{1}>0$, and $r_{3}>0$ iff $F<0$,

(c) the points are collinear iff $F=0$.

Lemma 2: If $n$ points $z_{1}, \ldots, z_{n}$ under cyclic pursuit are all collinear at some time $t_{1}$, then they are collinear for all $t<t_{1}$ and $t>t_{1}$.

Proof of Theorem 2 Consider the functions

$$
\begin{aligned}
& F_{i}(t)=\Im\left\{\left(\overline{z_{i}(t)-z_{0}}\right)\left(z_{i+1}(t)-z_{0}\right)\right\}, i=1, \ldots, n-1 \\
& F_{n}(t)=\Im\left\{\left(\overline{z_{n}(t)-z_{0}}\right)\left(z_{1}(t)-z_{0}\right)\right\} .
\end{aligned}
$$

By the definition of a counterclockwise star formation, $r_{i}(0)>0$ and $0<\alpha_{i}(0)<\pi$, where $\alpha_{i}, i=1,2, \ldots, n$ are as in Fig. 2. Hence, by Lemma $1, F_{i}(0)>0, \forall i$. We want to show that $F_{i}(t)>0$ for all $t$, implying $r_{i}(t)>0$ and $0<\alpha_{i}(t)<\pi$ for all $t$, by Lemma 1 . This means the points remain in a counterclockwise star formation.

Suppose by way of contradiction that some $F_{i}$, namely $F_{m}$, becomes zero at the first time $t_{1}$. We can select $m$ such that $F_{m+1}\left(t_{1}\right)>0$, for if all $F_{i}$ 's are zero at $t_{1}$, the points are all collinear, by Lemma 1 , which is a contradiction, by Lemma 2. Furthermore, for simplicity we renumber the indices if necessary so that $m+2 \leq n$. We have $F_{m}\left(t_{1}\right)=0 ; F_{m+1}\left(t_{1}\right)>0 ;$ and $F_{i}(t)>0, \forall t \in\left[0, t_{1}\right)$, $i=1,2, \ldots, n$. Taking the derivative along trajectories of

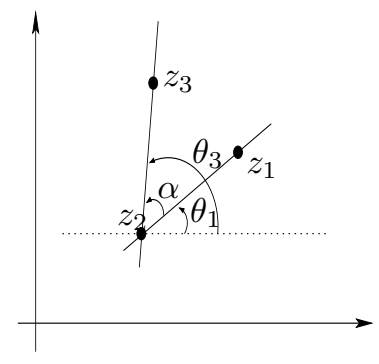

Fig. 3. Illustration for points $z_{1}, z_{2}, z_{3}$ and angles $\alpha, \theta_{1}, \theta_{3}$. 
the system $\dot{z}=A z$ and noting that $\dot{z}_{0}=0$, we have

$$
\begin{aligned}
\dot{F}_{m} & =\Im\left\{\overline{\dot{z}_{m}}\left(z_{m+1}-z_{0}\right)\right\}+\Im\left\{\overline{\left(z_{m}-z_{0}\right)} \dot{z}_{m+1}\right\} \\
& =-2 F_{m}+G_{m},
\end{aligned}
$$

where $G_{m}=\Im\left\{\overline{\left(z_{m}-z_{0}\right)}\left(z_{m+2}-z_{0}\right)\right\}$. Observe that $G_{m}=r_{m} r_{m+2} \sin \left(\alpha_{m}+\alpha_{m+1}\right)$.

By Lemma $1, F_{m}\left(t_{1}\right)=0$ implies that at $t_{1}$ either $r_{m}=0 ; r_{m+1}=0 ; \alpha_{m}=0$ and $r_{m}, r_{m+1}>0$; or $\alpha_{m}=\pi$ and $r_{m}, r_{m+1}>0$. We cannot have $r_{m+1}=0$ since $F_{m+1}\left(t_{1}\right)>0$. Condition $\alpha_{m}=\pi$ and $r_{m}, r_{m+1}>0$ is also impossible, since $\alpha_{i}\left(t_{1}\right) \geq 0, \forall i$, so all the points are either on the line formed by $z_{m+1}$ and $z_{m}$, a contradiction, or they are on or to one side of it, implying the centroid is not on the line formed by $z_{m+1}$ and $z_{m}$, also a contradiction. So consider the case that $\alpha_{m}=0$ and $r_{m}, r_{m+1}>$ 0 at $t_{1}$. Then $G_{m}\left(t_{1}\right)=\left(r_{m} / r_{m+1}\right) F_{m+1}\left(t_{1}\right)$. Hence $G_{m}\left(t_{1}\right)>0$. By continuity of $G_{m}$, there exists $0 \leq$ $t_{0}<t_{1}$ such that $G_{m}(t)>0$ for all $t \in\left[t_{0}, t_{1}\right]$. Also, by assumption, $F_{m}(t)>0$ for $t \in\left[0, t_{1}\right)$. Hence, $\dot{F}_{m}=-2 F_{m}+G_{m}>-2 F_{m}, t \in\left[t_{0}, t_{1}\right)$. Therefore $F_{m}(t)>\exp \left(-2\left(t-t_{0}\right)\right) F_{m}\left(t_{0}\right)>0, t \in\left[t_{0}, t_{1}\right)$. Ву continuity of $F_{m}, F_{m}\left(t_{1}\right)>0$, a contradiction.

Finally, consider the case that $r_{m}=0$ at $t=t_{1}$. Suppose that by a rotation of the coordinate system, if necessary, $z_{m+1}\left(t_{1}\right)=-r$, where $r=r_{m+1}\left(t_{1}\right)>0$. Also, to keep the notation simple, we renumber the points so that $m=n-1$. We have $\dot{z}_{m}\left(t_{1}\right)=z_{m+1}\left(t_{1}\right)-z_{m}\left(t_{1}\right)=-r$. Let $k<m$ be such that $r_{k}\left(t_{1}\right) \neq 0$ and $r_{j}\left(t_{1}\right)=0$ for $j=k+1, \ldots, m$. Such a $k$ exists, for if $r_{j}\left(t_{1}\right)=$ 0 for all $j \neq m+1$, then 0 is not the centroid, a contradiction. Now if $n=3$, we must have $\Im\left\{z_{k}\left(t_{1}\right)\right\}=0$; otherwise 0 is not the centroid. Then, the points are all collinear, a contradiction by Lemma 2 . If $n>3$, since $\alpha_{i}\left(t_{1}\right) \geq 0, \forall i$, we must have that $\Im\left\{z_{k}\left(t_{1}\right)\right\}>0$, for if $\Im\left\{z_{k}\left(t_{1}\right)\right\}=0$ then $\Im\left\{z_{i}\left(t_{1}\right)\right\} \leq 0, \forall i$, implying the points are all collinear or the centroid is not 0 , both contradictions. The geometry of the situation is depicted in Fig. 4. Let $\theta_{k}\left(t_{1}\right)=\delta>0$. By continuity of $\theta_{k}$, there exists $t_{0}<t_{1}$ such that for $t \in\left[t_{0}, t_{1}\right], \theta_{k}(t)>\delta / 2$. Since the points are in a counterclockwise star formation until $t_{1}$ we have that $\theta_{m}(t)>\theta_{k}(t)$ for $t \in\left[t_{0}, t_{1}\right)$. By definition $z_{m}\left(t_{1}\right)-z_{m}\left(t_{1}-h\right)=h \dot{z}_{m}\left(t_{1}\right)+O(h)$. We obtain $\Re\left\{z_{m}\left(t_{1}-h\right)\right\}=r h+O(h)$ and $\Im\left\{z_{m}\left(t_{1}-h\right)\right\}=O(h)$.

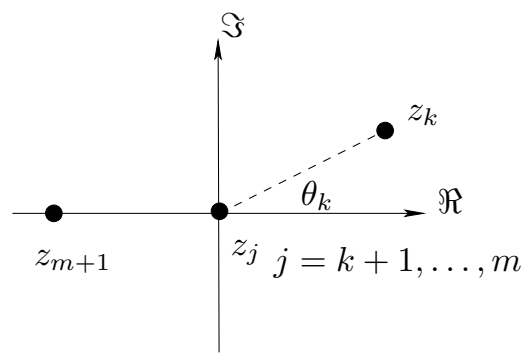

Fig. 4. Illustration for points at $t_{1}$.
Thus, for $h>0$ sufficiently small $\Re\left\{z_{m}\left(t_{1}-h\right)\right\}>0$ and we know $\Im\left\{z_{m}\left(t_{1}-h\right)\right\}>\Re\left\{z_{m}\left(t_{1}-h\right)\right\} \tan \delta / 2$, with $0<\delta / 2<\pi / 2$. Combining these facts we obtain $O(h)>$ $(r h+O(h)) \tan \delta / 2$. Dividing by $h$ and taking the limit as $h \rightarrow 0$ we obtain $\lim _{h \rightarrow 0}|O(h) / h|>r \tan \delta / 2>0$, a contradiction.

The reader is referred to [18] for further interesting results on formation evolution under cyclic pursuit.

\section{SENSORS With A LiMITED FIELD OF VIEW: UNDIRECTED-GRAPH CASE}

In this section, adapting the setup in [7] we study a different control strategy that is motivated by Reynolds' cohesion steering strategy. Suppose as before that there are $n$ autonomous agents represented by points in the complex plane and numbered 1 through $n$; the agents don't need to know the labels. Each agent has a sensor with a limited field of view, in that it can see and know the relative positions of only those agents that are within some distance of itself, called neighbor agents. Let $N_{i}(t)$ denote the set of labels of agent $i$ 's neighbor agents at time $t$. Reynolds' cohesion strategy is for agent $i$ to steer towards the average of the neighbor agents' directions. For a technical reason (namely, to get a common Lyapunov function), we consider where agent $i$ steers towards the sum of the neighbor agents' directions. Thus the kinematic equation is

$$
\dot{z}_{i}(t)=\sum_{j \in N_{i}(t)}\left[z_{j}(t)-z_{i}(t)\right], \quad i=1, \ldots, n .
$$

We use an undirected graph $\mathcal{G}$ with vertex set $\left\{z_{1}, z_{2}, \ldots, z_{n}\right\}$ to describe the sensor relationship among agents: $\left(z_{i}, z_{j}\right)$ is an edge iff agents $z_{i}$ and $z_{j}$ are within sensor range of each other (all the sensors are assumed to have the same range). We call this the sensor graph. The sensor relationship changes over time, so the sensor graph changes too. Let $\left\{\mathcal{G}_{p}: p \in \mathcal{P}\right\}$ denote the class of all possible undirected graphs defined on $n$ vertices. Corresponding to each graph $\mathcal{G}_{p}$, let $J_{p}$ denote the (symmetric) adjacency matrix, let $D_{p}$ denote the diagonal matrix whose $i$ th diagonal element is the valence of vertex $i$, and define $A_{p}=J_{p}-D_{p}$ (the negative of the Laplacian).

At time $t$, let the sensor graph be $\mathcal{G}_{p(t)}$ and let the corresponding matrix be $A_{p(t)}$. The overall system is then

$$
\dot{z}(t)=A_{p(t)} z(t)
$$

where $z(t)$ is the position vector. The signal $p(t)$ switches among a finite number of values as $t$ progresses. It is assumed that chattering doesn't occur, that is, that $p(t)$ switches a finite number of times in every finite time interval. Then (2) has a well-defined solution.

Our goal is to show that, for a class of switching signals $p(t)$ and a class of initial configurations of the agents, all agents converge to the same point (i.e., the centroid). We need an assumption to prove this (the same assumption as in [7]), namely, that the sensor graph is always connected. 
Theorem 3: With regard to system (2), assume $\mathcal{G}_{p(t)}$ is connected for every $t \geq 0$. Then the centroid of the points $z_{1}(t), \ldots, z_{n}(t)$ is stationary and every $z_{i}(t)$ converges to this centroid.

Proof If $\mathcal{G}_{p}$ is connected, $A_{p}$ has the properties that every row sum is equal to 0 , the diagonal elements are less than 0 , and the other elements are non-negative. Thus, letting $a_{i j}$ denote the $i j$ th element of $A_{p}$, we have $-a_{i i}=$ $\sum_{j \neq i} a_{i j}>0, \forall i$. Hence, from Gerschgorin's theorem, the eigenvalues of $A_{p}$ are in the set $\{\lambda: \operatorname{Re} \lambda<0$ or $\lambda=0\}$. In particular, the nonzero eigenvalues have negative real parts.

Next we observe, again when $\mathcal{G}_{p}$ is connected, that $A_{p}$ has a unique zero eigenvalue, i.e., rank $A_{p}=n-1$. To see this, note that $D_{p}$ is nonsingular and define a new matrix

$$
\tilde{A}_{p}=D_{p}^{-1} A_{p}=B_{p}-I, \quad B_{p}:=D_{p}^{-1} J_{p} .
$$

The associated graph $\mathcal{G}\left(J_{p}\right)$ is connected, and so is the associated graph $\mathcal{G}\left(B_{p}\right)$. Therefore, $B_{p}$ is irreducible. By the Perron-Frobenius theorem, $B_{p}$ has a unique largest real eigenvalue $\lambda_{1}$, and the other eigenvalues $\lambda_{i}, i=$ $2, \ldots, n$, satisfy $\operatorname{Re} \lambda_{i}<\lambda_{1}$. So $\tilde{A}_{p}$, with eigenvalues $\left\{\lambda_{1}-1, \ldots, \lambda_{n}-1\right\}$, has a unique largest real one. Using Gerschgorin's theorem again, we know that the eigenvalues of $\tilde{A}_{p}$ are in the set $\{\lambda: \operatorname{Re} \lambda<0$ or $\lambda=0\}$ and also we know that $\tilde{A}_{p}$ has a zero eigenvalue. Consequently, $\tilde{A}_{p}$ has a unique largest eigenvalue $\lambda_{\max }=0$, i.e., rank $A_{p}=$ rank $\tilde{A}_{p}=n-1$.

For every $t, A_{p(t)}$ has $\lambda=0$ as an eigenvalue of multiplicity 1 and the vector $v$ of 1 's is a common eigenvector. Thus $\mathcal{E}_{0}=\operatorname{span}\{v\}$ is a common eigenspace and its orthogonal complement, $\mathcal{E}_{1}$, is the sum of all the other eigenspaces, for all $t$. The trajectory looks like $z(t)=a v+w(t), w(t) \in \mathcal{E}_{1}$ and it remains to show that $w(t) \rightarrow 0$.

Since $w(t)=z(t)-a v$ and $A_{p(t)} v=0$, we have $\dot{w}(t)=\dot{z}(t)=A_{p(t)} z(t)=A_{p(t)} w(t)$. We know that for any $w(0) \in \mathcal{E}_{1}$, the solution $w(t) \in \mathcal{E}_{1}, \forall t \geq 0$. In other words, $\mathcal{E}_{1}$ is a positively invariant set for the system $\dot{w}(t)=A_{p(t)} w(t)$. Choose the Lyapunov function $V(w)=$ $(1 / 2) w^{T} w$. Take the derivative of $V(w(t))$ along the solution of $\dot{w}(t)=A_{p(t)} w(t): \dot{V}(w(t))=w^{T}(t) A_{p(t)} w(t)$. Then, $\dot{V}(w(t))=w^{T}(t) A_{p(t)} w(t) \leq-W(w(t))$, where $W(w):=-\left(\max \lambda_{p 1}\right) w^{T} w, \lambda_{p 1}$ is the largest nonzero eigenvalue of $A_{p}^{p}$, and the max is over all $p$ for which the sensor graph is connected. Thus $W(w)>0, \forall w \in \mathcal{E}_{1}-\{0\}$ and $W(0)=0$. Therefore, by the Lyapunov stability theorem for non-autonomous systems [8], the trajectory starting in $\mathcal{E}_{1}$ converges to 0 .

We simulated twenty mobile autonomous agents. In Fig. 5, the sensor range was adequate; in Fig. 6 it was not.

\section{Sensors with a Limited Field of View: DIRECTED-GRAPH CASE}

In the previous section, we considered where each autonomous agent can sense only those agents within a disk of pre-specified radius centered about itself. What if the

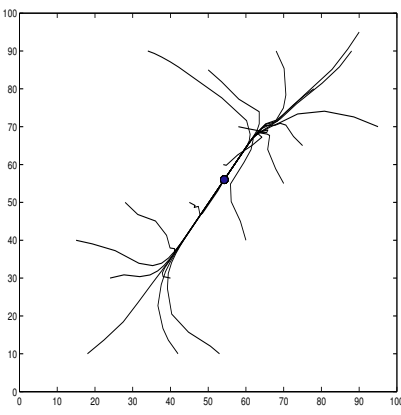

Fig. 5. Sensor range is 30 .

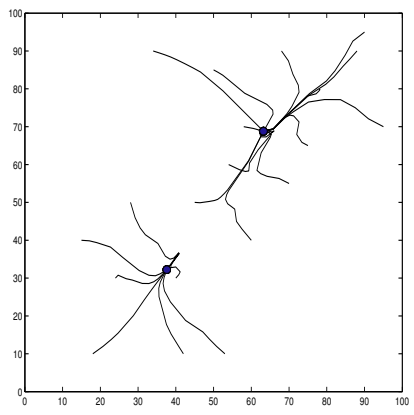

Fig. 6. Sensor range is 25 . camera doesn't have disk-like visibility but a cone-like field of view? In this section, we will present a general result about convergence of a group of agents. Again, we borrow a technique from [7], namely, use of Wolfowitz's theorem.

We consider the local control strategy where each agent pursues the centroid of the subgroup of sensed agents at time t. Let $n_{i}(t)$ denote the cardinality of $N_{i}(t)$ (the number of agents sensed by agent $i$ at time $t$ ). Then the $i$-th agent's kinematic equation is

$$
\dot{z}_{i}(t)=\left\{\begin{array}{cc}
\frac{1}{n_{i}(t)} \sum_{j \in N_{i}(t)}\left\{z_{j}(t)-z_{i}(t)\right\}, & n_{i}(t) \neq 0, \\
0, & n_{i}(t)=0 .
\end{array}\right.
$$

The sensor graph is now directed. Let $\left\{\mathcal{G}_{p}: p \in \mathcal{P}\right\}$ denote all possible directed graphs $\mathcal{G}_{p}$ defined on $n$ vertices. Corresponding to each directed graph $\mathcal{G}_{p}$, let $J_{p}$ denote the adjacency matrix, let $D_{p}$ denote the diagonal matrix whose $i$ th diagonal element is the number of directed edges from vertex $i$ to others, and let $U_{p}$ denote the diagonal matrix whose $i$ th diagonal element is the reciprocal of the $i$ th diagonal element of matrix $D_{p}$ if it is not zero, and 0 if it is zero. Define $A_{p}=U_{p}\left(J_{p}-D_{p}\right)$. Then the model is (2).

Assuming connectedness of the graph for all $t$ is too strong. Rather let $\mathcal{G}_{p(t)}$ be disconnected some times and connected other times. We denote by $\left\{\mathcal{G}_{p}: p \in \mathcal{Q}\right\}$ all strongly connected graphs.

Theorem 4: Assume the switching signal $p(t)$ is piecewise constant and there exists a positive $T$ so that $p(t) \in \mathcal{Q}$ at least once in each time interval of length $T$. Then each $z_{i}(t)$ converges to the same point $a$, where $a$ depends only on $z(0)$ and $p(t)$.

Some preliminaries are required for the proof. A nonnegative matrix has all entries nonnegative; a square real matrix is row stochastic if it is nonnegative and its row sums all equal 1.

Theorem 5: [23] Let $\left\{P_{1}, P_{2}, \ldots\right\}$ be a finite or infinite set of row stochastic matrices satisfying $0 \leq \lambda\left(P_{i}\right) \leq$ $\beta<1$. Then for each infinite sequence, $P_{k_{1}}, P_{k_{2}}, \ldots$, there exists a row vector $c$ such that $\lim _{j \rightarrow \infty} P_{k_{j}} P_{k_{j-1}} \cdots P_{k_{1}}=\mathbf{1} c$, where $1:=\left[\begin{array}{llll}1 & 1 & \cdots & 1\end{array}\right]^{T}$.

This version of Theorem 5 is somewhat stronger than Wolfowitz's original version, in that an infinite number of 
matrices are allowed. The proof of the modified one is given in [10].

Proof of Theorem 4 Suppose $p$ changes its value at time instants $t_{0}=0<t_{1}<t_{2}<\cdots$. If there are actually only finitely many switches, the final at $t_{m}$, artificially define $t_{m+j}=t_{m}+j b, j=1,2, \ldots$, where $b>0$. So the time $\mathbb{R}^{+}$can be divided into an infinite number of time intervals $\left[t_{0}, t_{1}\right),\left[t_{1}, t_{2}\right), \ldots$. Since $p(t)$ is piecewise constant, there exist $t_{\text {max }}, t_{\min }>0$ such that $t_{\text {min }} \leq t_{i+1}-t_{i} \leq t_{\max }$. Also, $A_{p(t)}=A_{p\left(t_{i}\right)}, \forall t \in\left[t_{i}, t_{i+1}\right)$. From (2)

$z(t)=\mathrm{e}^{A_{p\left(t_{i}\right)}\left(t-t_{i}\right)} \mathrm{e}^{A_{p\left(t_{i-1}\right)}\left(t_{i}-t_{i-1}\right)} \cdots \mathrm{e}^{A_{p\left(t_{0}\right)}\left(t_{1}-t_{0}\right)} z(0)$.

Define $\Phi\left(t, t_{i}\right)=\mathrm{e}^{A_{p\left(t_{i}\right)}\left(t-t_{i}\right)}, t \in\left[t_{i}, t_{i+1}\right]$, so

$z(t)=\Phi\left(t, t_{i}\right) \Phi\left(t_{i}, t_{i-1}\right) \cdots \Phi\left(t_{1}, t_{0}\right) z(0), \quad t \in\left[t_{i}, t_{i+1}\right]$.

For the given $T$, generate a subsequence $\left\{t_{m_{j}}\right\}$ of $\left\{t_{i}\right\}$ as follows: (1) Set $t_{m_{0}}=t_{0}=0$. (2) If $T \in\left(t_{i-1}, t_{i}\right]$, set $t_{m_{1}}=t_{i}$. (3) If $t_{m_{1}}+T \in\left(t_{i-1}, t_{i}\right]$, set $t_{m_{2}}=t_{i}$. And so on. Then taking $T_{j}=t_{m_{j}}$, we have

$$
\begin{aligned}
z\left(T_{1}\right) & =\Phi\left(t_{m_{1}}, t_{m_{1}-1}\right) \Phi\left(t_{m_{1}-1}, t_{m_{1}-2}\right) \cdots \Phi\left(t_{1}, t_{0}\right) z(0) \\
& =: \Psi_{1} z(0), \\
z\left(T_{2}\right) & =\Phi\left(t_{m_{2}}, t_{m_{2}-1}\right) \cdots \Phi\left(t_{m_{1}+1}, t_{m_{1}}\right) z\left(T_{1}\right) \\
& =: \Psi_{2} z\left(T_{1}\right)=\Psi_{2} \Psi_{1} z(0)
\end{aligned}
$$

and so on so that $z\left(T_{j}\right)=\Psi_{j} \Psi_{j-1} \cdots \Psi_{1} z(0)$.

We now show that $\lim _{j \rightarrow \infty} z\left(T_{j}\right)=a \mathbf{1}$, for which suffices to show that $\lim _{j \rightarrow \infty} \Psi_{j} \cdots \Psi_{1}=\mathbf{1} c$, where $c$ is a row vector. (Then $a=c z(0)$.) The matrix $A_{p}, p \in \mathcal{P}$, satisfies (a) $A_{p_{i i}} \leq 0, \forall i$; (b) $A_{p_{i j}} \geq 0, \forall i \neq j$; (c) $\sum_{j} A_{p_{i j}}=0$, $\forall i$. Hence by Theorem 29, p. 151, of [6], we know that $\Phi\left(t_{i+1}, t_{i}\right)=\mathrm{e}^{A_{p\left(t_{i}\right)}\left(t_{i+1}-t_{i}\right)}$ is row stochastic and the diagonal elements are lower-bounded by a positive value $\epsilon_{1}$ since $\mathcal{P}$ is a finite set and $t_{i+1}-t_{i}$ is in the closed interval $\left[t_{\min }, t_{\max }\right]$. Furthermore, for $p \in \mathcal{Q}$ (i.e., $\mathcal{G}_{p}$ is strongly connected), $A_{p}=U_{p}\left(J_{p}-D_{p}\right)=U_{p} J_{p}-I$, and the matrix $V_{p}:=U_{p} J_{p}$ is irreducible. Hence,

$$
\begin{aligned}
\Phi\left(t_{i+1}, t_{i}\right) & =\mathrm{e}^{A_{p\left(t_{i}\right)}\left(t_{i+1}-t_{i}\right)}=\mathrm{e}^{-I\left(t_{i+1}-t_{i}\right)} \mathrm{e}^{V_{p\left(t_{i}\right)}\left(t_{i+1}-t_{i}\right)} \\
& =\mathrm{e}^{-\left(t_{i+1}-t_{i}\right)}\left\{I+V_{p\left(t_{i}\right)}\left(t_{i+1}-t_{i}\right)+\cdots\right\} .
\end{aligned}
$$

From the above expression, we can see that if $p \in \mathcal{Q}$, each element of $\Phi\left(t_{i+1}, t_{i}\right)$ is lower-bounded by a positive value $\epsilon_{2}$. That is, $\Phi\left(t_{i+1}, t_{i}\right) \geq \epsilon_{2} M, i=0,1,2, \ldots$, where $M$ is a matrix with all elements 1 and the inequality is componentwise.

If $p(t) \in \mathcal{Q}$ happens at least once in each time interval of length $T$, then at least one of the transition matrices $\Phi$ in the matrix product $\Psi_{j}=$ $\Phi\left(t_{m_{j}}, t_{m_{j}-1}\right) \cdots \Phi\left(t_{m_{j-1}+1}, t_{m_{j-1}}\right)$ is row stochastic with each element greater than $\epsilon_{2}$, and the others are row stochastic matrices with each diagonal element greater than $\epsilon_{1}$. In each time interval of length $T$, there are a finite number of switching times. Let's say the maximum number of switching times is $K$ for every time interval of length
$T$. As a result, $\Psi_{j}, j=1,2, \ldots$ is row stochastic and each element is lower-bounded by $\epsilon_{2} \epsilon_{1}^{K-1}$. Thus, $0 \leq \lambda\left(\Psi_{j}\right) \leq$ $1-n \epsilon_{2} \epsilon_{1}^{K-1}<1, j=1,2, \ldots$. Therefore, by Theorem 5 , $\lim _{j \rightarrow \infty} \Psi_{j} \cdots \Psi_{1}=\mathbf{1} c$, where $c$ is a row vector.

Now we have to look at the times between the instants $\left\{0, T_{1}, T_{2}, \ldots\right\}$. For $t \in\left[T_{j}, T_{j+1}\right]$,

$$
z(t)=\Phi\left(t, t_{m_{j}+k}\right) \cdots \Phi\left(t_{m_{j}+1}, t_{m_{j}}\right) z\left(T_{j}\right)=: \widetilde{\Phi} z\left(T_{j}\right),
$$

where $\widetilde{\Phi}$ is a row stochastic matrix since $\Phi\left(t, t_{m_{j}+k}\right), \ldots$, $\Phi\left(t_{m_{j}+1}, t_{m_{j}}\right)$ are all row stochastic. Hence, $z_{i}(t)$ is in the convex hull of the set $\left\{z_{i}\left(T_{j}\right): i=1,2, \ldots, n\right\}$. It follows by a simple convexity argument that

$$
\max _{i_{1}, i_{2}}\left\|z_{i_{1}}(t)-z_{i_{2}}(t)\right\| \leq \max _{q_{1}, q_{2}}\left\|z_{q_{1}}\left(T_{j}\right)-z_{q_{2}}\left(T_{j}\right)\right\| .
$$

We conclude that $\lim _{t \rightarrow \infty} z(t)=a \mathbf{1}$, where $a$ is a complex number depending only on $z(0)$ and $p(t)$.

Theorem 4 is related to Theorem 2 in [7], with the following main differences: The setup in Theorem 2 of [7] is discrete-time whereas ours is continuous-time; the sensor graph in Theorem 2 of [7] is undirected whereas our's is directed; the original theorem of Wolfowitz, with finitely many matrices, is used in Theorem 2 of [7] (indeed, the authors in [7] say: "The finiteness of the set $M_{1}, M_{2}, \ldots, M_{m}$ is crucial to Wolfowitz's proof.") whereas we needed a version with infinitely many matrices.

Fig. 7 shows a simulation for $n=5$. Each agent has a cone-like field of view with infinite radius and 90 degree angle. Each agent is programmed to rotate its view angle $90^{\circ}$ clockwise if there are no other agents in its field of

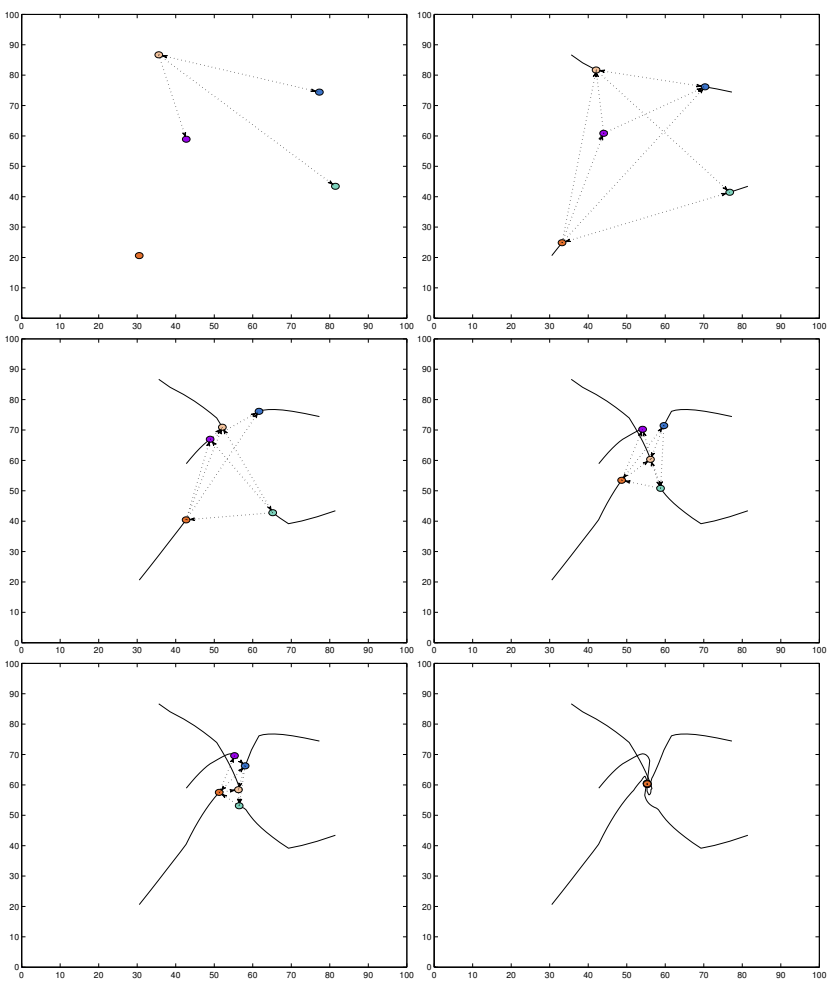

Fig. 7. Trajectories and the sensor graphs as the system evolves. 
view. From Fig. 7, we can see that the initial sensor graph is not strongly connected. As the system evolves, the sensor graph might be strongly connected for some time and then disconnected for some other time.

As a final comment, it's not necessary for each agent to pursue the centroid of the subgroup of sensed agents to achieve convergence. Considering a more general control strategy based on a general linear combination of the subgroup, we have the $i$-th agent's kinematic equation

$$
\dot{z}_{i}(t)=\sum_{j \in N_{i}(t)} a_{i j}(t)\left[z_{j}(t)-z_{i}(t)\right], \quad a_{i j}>0,
$$

or in vector form $\dot{z}(t)=\widetilde{A}(t) z(t)$, where element $(i, i)$ of $\widetilde{A}(t)$ is $-\sum_{j \in N_{i}(t)} a_{i j}(t)$ and element $(i, j)$ is $a_{i j}(t)$ if $j \in N_{i}(t)$ and 0 otherwise.

Theorem 4 still applies to this general system. Notice that cyclic pursuit is a special case of (4), where $a_{i j}(t)=1$ and $N_{i}(t)=\{i+1\}, i=1,2, \ldots, n-1, N_{n}(t)=\{1\}$; and (3) is a special case of (4), where $a_{i j}(t)=1$ and $N_{i}(t)$ contains the labels of agent $i$ 's neighbor agents at time $t$.

\section{CONClusion}

Control of systems consisting of several autonomous agents that are intended to perform a coordinated task is currently an important and challenging field of research. This is due to the broad range of applications of multiagent systems in space missions, operations in hazardous environments, and military operations. In this paper, we studied three formation strategies for coordinated control of groups of mobile autonomous agents modeled as point masses with full actuation. Each agent relies only on locally available information, namely, the relative locations of a sensed subgroup of agents. Global information and communication are not required. Instead, local sensors (perhaps vision) can be used to generate effective global group behavior.

\section{REFERENCES}

[1] H. Ando, I. Suzuki, and M. Yamashita, "Formation and agreement problems for synchronous mobile robots with limited visibility," in Proc. IEEE Int. Symp. on Intelligent Control, 1995, pp. 453-460.

[2] A.M. Bruckstein, N. Cohen, and A. Efrat, "Ants, crickets, and frogs in cyclic pursuit," Center for Intelligent Systems Report 9105, Technion, Haifa, Israel, 1991.

[3] Q. Chen and J. Y. S. Luh, "Coordination and control of a group of small mobile robots," in IEEE Int. Conf. on Robotics and Automation, 1994, pp. 2315-2320.

[4] J. Feddema, C. Lewis, and D. Schoenwald, "Decentralized control of cooperative robotic vehicles: theory and application," IEEE Trans. Robot. Automat., vol. 18, 2002, pp. 852-864.

[5] J. Fredslund and M. J. Mataric, "A general algorithm for robot formation using local sensing and minimal communication," IEEE Trans. Robot. Automat., vol. 18, 2002, pp. 837-846.
[6] D. Freedman, Markov Chains, Springer-Verlag, 1983.

[7] A. Jadbabaie, J. Lin, and A.S. Morse, "Coordination of groups of mobile autonomous agents using nearest neighbor rules," IEEE Trans. Auto. Control, vol. 48, 2003, pp. 988-1001.

[8] H.K. Khalil, Nonlinear Systems, Prentice Hall, 1996.

[9] N. E. Leonard and E. Fiorelli, "Virtual leaders, artificial potentials and coordinated control of groups," in Proc. IEEE Conf. on Decision and Control, 2001, pp. 2968-2973.

[10] Z. Lin, M. Broucke, and B. Francis, "Local control strategies for groups of mobile autonomous agents," www.control.utoronto.ca/people/profs/francis/publications.html

[11] Y. Liu, K. Passino, and M. Polycarpou, "Stability analysis of $M$-dimensional asynchronous swarms with a fixed communication topology," IEEE Trans. Auto. Control, vol. 48, 2003, pp. 76-95.

[12] J. Marshall, M. Broucke, and B. Francis, "A pursuit strategy for wheeled-vehicle formations," in IEEE Conf. on Decision and Control, 2003.

[13] Y. Oasa, I. Suzuki, and M. Yamashita, "A robot distributed convergence algorithm for autonomous mobile robots," in IEEE Int. Conf. on Systems, Man, and Cybernetics, 1997, pp. 287-292.

[14] Y. Oasa, I. Suzuki, and M. Yamashita, "Distributed memoryless point convergence algorithm for mobile robots with limited visibility," IEEE Trans. Robot. Automat., vol. 15, 1999, pp. 818-828.

[15] R. Olfati-Saber and R.M. Murray, "Distributed cooperative control of multiple vehicle formations using structural potential functions," in IFAC World Congress, 2002.

[16] C. Reynolds, "Flocks, birds, and schools: a distributed behavioral model," Computer Graphics, vol. 21, 1987, pp. 25-34.

[17] C. Reynolds, www.red3d.com/cwr/boids/

[18] T. Richardson, "Stable polygons of cyclic pursuit," Annals of Mathematics and Artificial Intelligence, vol. 31, 2001, pp. 147-172.

[19] K. Sugihara and I. Suzuki, "Distributed motion coordination of multiple mobile robots," in Proc. IEEE Int. Symp. on Intelligent Control, 1990, pp. 138-143.

[20] I. Suzuki and M. Yamashita, "Distributed anonymous mobile robots: formation of geometric patterns," SIAM J. Comput., vol. 28, 1999, pp. 1347-1363.

[21] P. K. C. Wang, "Navigation strategies for multiple autonomous mobile robots moving in formation," in IEEE/RSJ Int. Workshop on Intelligent Robots and Systems, 1989, pp. 486-493.

[22] P. K. C. Wang, "Navigation strategies for multiple autonomous mobile robots moving in formation," $J$. of Robotic Systems, vol. 8, 1991, pp. 177-195.

[23] J. Wolfowitz, "Products of indecomposable, aperiodic, stochastic matrices," Proc. American Mathematical Society, vol. 14, 1963, pp. 733-737. 